\title{
A LIMIT ON THE LOEWY LENGTH OF THE ENDOMORPHISM RING OF A MODULE OF FINITE LENGTH
}

\author{
SVERRE O. SMALO
}

\begin{abstract}
Let $M$ be a module of finite length over an arbitrary ring $A$. Let $S_{1}$, $S_{2}, \ldots, S_{m}$ be the nonisomorphic simple composition factors of $M$ and let $[M]=$ $n_{1}\left[S_{1}\right]+n_{2}\left[S_{2}\right]+\cdots+n_{m}\left[S_{m}\right]$ denote that $S_{i}$ occurs $n_{i}$ times in a composition series for $M$. As a generalization of Schur's lemma we have the following wellknown result: If $M$ is nonzero and all the $n_{i}$ are equal to one then the Loewy length of the endomorphism ring of $M$ is one. We will generalize this fact showing that in general the Loewy length of the endomorphism ring of $M$ is less than or equal to the maximum of the $n_{i}, i=1, \ldots, m$.
\end{abstract}

Throughout this note $M$ will denote a module of finite length over an arbitrary ring $A$. We will denote by $S_{1}, S_{2}, \ldots, S_{m}$ the set of nonisomorphic composition factors of $M$, and we will let $[M]=n_{1}\left[S_{1}\right]+\cdots+n_{m}\left[S_{m}\right]$ denote that $S_{i}$ occurs $n_{i}$ times as a composition factor in a composition series of $M$. It is well known that $\operatorname{rad}(\operatorname{End}(M))$, the radical of $\operatorname{End}(M)$, is nilpotent [1, p. 324]. We will let $\operatorname{LL}(\operatorname{End}(M))$ denote the Loewy length of such a ring, i.e. $\operatorname{LL}(\operatorname{End}(M))$ is the integer $n$ such that $\operatorname{rad}^{n}(\operatorname{End}(M))=0$, but $\operatorname{rad}^{n-1}(\operatorname{End}(M)) \neq 0$. Further, we will let $\operatorname{Ker} f$ and $\operatorname{Im} f$ denote the kernel and the image of a map $f$ respectively. A classical result in this situation is Schur's lemma and a direct generalization of it is the following easily verified result: Let $M$ be a module of finite length over a ring $A$ and assume $[M]=\left[S_{1}\right]+\cdots+\left[S_{m}\right]$, then $\operatorname{LL}(\operatorname{End}(M))=1$.

We are going to generalize this to obtain the following general result.

THEOREM. Let $M$ be a nonzero module of finite length over $a$ ring $A$ and assume $[M]=n_{1}\left[S_{1}\right]+\cdots+n_{m}\left[S_{m}\right]$. Then

$$
\operatorname{LL}(\operatorname{End}(M))<\max \left\{n_{i} \mid i=1, \ldots, m\right\} \text {. }
$$

To prove this Theorem we will start out with a Lemma in which we need the following notion.

A subset $B$ of a ring $A$ is called nilpotent in case there is an integer $t>0$ such that $x_{1} x_{2} \ldots x_{t}=0$ for all sequences $x_{1}, x_{2}, \ldots, x_{t}$ in $B$.

LEMMA. Let $M$ be a module of finite length over a ring $A$ and assume $[M]=n_{1}\left[S_{1}\right]$ $+\cdots+n_{m}\left[S_{m}\right]$ with all $n_{i} \neq 0$.

(i) Let $\left\{f_{i}\right\}_{i \in I}$ be a family of maps in $\operatorname{End}(M)$ which is nilpotent. Then $\left[\cap_{i} \operatorname{Ker} f_{i}\right]$ $=n_{1}^{\prime}\left[S_{1}\right]+\cdots+n_{m}^{\prime}\left[S_{m}\right]$ with all $n_{i}^{\prime} \neq 0, i=1, \ldots, m$.

Received by the editors February 11, 1980.

AMS (MOS) subject classifications (1970). Primary 16A64, 16A22, 16A46.

(C) 1981 American Mathematical Society $0002-9939 / 81 / 0000-0051 / \$ 01.75$ 
(ii) $[\cap\{\operatorname{Ker} f \mid f \in \operatorname{rad}(\operatorname{End}(M))\}]=n_{1}^{\prime \prime}\left[S_{1}\right]+\cdots+n_{m}^{\prime \prime}\left[S_{m}\right]$ with all $n_{i}^{\prime \prime} \neq 0$, $i$ $=1, \ldots, m$.

(iii) $[M / \Sigma\{\operatorname{Im} f \mid f \in \operatorname{rad}(\operatorname{End}(M))\}]=n_{1}^{\prime \prime \prime}\left[S_{1}\right]+\cdots+n_{m}^{\prime \prime \prime}\left[S_{m}\right]$ with all $n_{i}^{\prime \prime \prime} \neq$ $0, i=1, \ldots, m$.

Proof. (i) Assume $\left\{f_{i}\right\}_{i \in I}$ is a nilpotent family of maps in $\operatorname{End}(M)$ and assume $\cap\left\{\operatorname{Ker} f_{i} \mid i \in I\right\}$ has a composition series with no composition factor isomorphic to $S_{i}$ for some $i \in\{1, \ldots, m\}$. Since $M$ is artin there exists a finite set $\left\{f_{1}, \ldots, f_{k}\right\}$ in $\left\{f_{i}\right\}_{i \in I}$ such that $\bigcap_{j=1}^{k} \operatorname{Ker} f_{j}=\cap\left\{\operatorname{Ker} f_{i} \mid i \in I\right\}$. Consider now the map $h_{1}$ : $M \rightarrow M^{k}$ with coordinates $f_{j}, j=1, \ldots, k$. Here $M^{k}$ denotes a direct sum of $k$ copies of $M$. Then $\operatorname{Ker} h_{1}=\bigcap_{j=1}^{k} \operatorname{Ker} f_{j}$. Further let $h_{2}:\left(M^{k}\right) \rightarrow\left(M^{k}\right)^{k}$ be the map with coordinates $h_{1}$. Then $\operatorname{Ker} h_{2}=\left(\operatorname{Ker} h_{1}\right)^{k}=\left(\cap_{j=1}^{k} \operatorname{Ker} f_{j}\right)^{k}$. This shows that also $\operatorname{Ker} h_{2}$ has a composition series which contains no composition factor isomorphic to $S_{i}$. Therefore the same holds for Ker $h_{2} h_{1}$. Now by an easy induction argument we obtain for each $p$ a map $h_{p}: M^{k^{p-1}} \rightarrow M^{k^{p}}$ such that all coordinates are among the $f_{j}, j=1, \ldots, k$, and such that $\operatorname{Ker} h_{p} h_{p-1} \ldots h_{1}$ has no composition series which contains a composition factor isomorphic to $S_{i}$. But $\operatorname{Ker} h_{p} \ldots h_{1}$ $=\cap \operatorname{Ker} f_{j_{p}} f_{j_{p-1}} \ldots f_{j_{1}}$ where the intersection is taken over all ordered $p$-tuples of the maps $f_{1}, f_{2}, \ldots, f_{k}$. By assumption the set $\left\{f_{i}\right\}_{i \in I}$ is nilpotent and therefore there exists a $p$ such that all these compositions are zero. But then $\cap \operatorname{Ker} f_{p} f_{p-1} \ldots f_{1}=M$ and hence has to have a composition series which contains a composition factor isomorphic to $S_{i}$. This is a contradiction. Therefore $\cap\left\{\operatorname{Ker} f_{i} \mid i \in I\right\}$ has a composition series which for each $i=1, \ldots, m$ contains a composition factor isomorphic to $S_{i}$. This completes the proof of (i).

(ii) This follows from (i) since $\operatorname{rad}(\operatorname{End}(M))$ is nilpotent.

(iii) One obtains this result by dualizing the proof of (i).

We are now in a position where we can prove the Theorem. For each $j$ we claim that $M_{j}=\cap\left\{\operatorname{Ker} f \mid f \in \operatorname{rad}^{j}(\operatorname{End}(M))\right\}$ has the property that $\left[M_{j}\right]=n_{1, j}\left[S_{1}\right]$ $+\cdots+n_{m, j}\left[S_{m}\right]$ where $n_{i, j} \geqslant \min \left\{n_{i}, j\right\}$. This will be proved by induction using the Lemma. By the Lemma this holds for $j=1$. Assume now that the claim is true for $j<k$ and consider $M_{k+1}=\bigcap\left\{\operatorname{Ker} f \mid f \in \operatorname{rad}^{k+1}(\operatorname{End}(M))\right\}$ and let $\left[M_{k+1}\right]=$ $n_{1, k+1}\left[S_{1}\right]+\cdots+n_{m, k+1}\left[S_{m}\right]$. Suppose there is an $i \in\{1, \ldots, m\}$ such that $n_{i, k+1}<\min \left\{n_{i}, k+1\right\}$. It is easy to see that $n_{i, k+1} \geqslant n_{i, k}$ and by the induction hypothesis $n_{i, k} \geqslant \min \left\{n_{i}, k\right\}$. Hence $n_{i, k+1}=n_{i, k}=k$ and $n_{i}>k$. This means that the module $M / M_{k}$ has a composition series with a composition factor isomorphic to $S_{i}$ and that $M_{k+1} / M_{k}$ has a composition series with no composition factor isomorphic to $S_{i}$. But

$$
M_{k+1}=\bigcap\left\{g^{-1}\left(M_{k}\right) \mid g \in \operatorname{rad}(\operatorname{End}(M))\right\}
$$

and

$$
M_{k+1} / M_{k}=\cap\left\{\operatorname{Ker} f^{\prime} \mid f \in \operatorname{rad}(\operatorname{End}(M))\right\}
$$

where $f^{\prime}: M / M_{k} \rightarrow M / M_{k}$ is the map induced by $f$. Now the family $\left\{f^{\prime}\right\}$ of maps in $\operatorname{End}\left(M / M_{k}\right)$ induced by the maps in $\operatorname{rad}(\operatorname{End}(M))$ is a nilpotent family of maps. We can now apply the Lemma to get a contradiction. This completes the proof of the claim. It is easy to see that $\operatorname{LL}(\operatorname{End}(M))$ is equal to the least integer $t$ such that 
$M_{t}=M$. Let $q=\max \left\{n_{i} \mid i=1, \ldots, m\right\}$ and consider $\left[M_{q}\right]=n_{1, q}\left[S_{1}\right]$ $+\cdots+n_{m, q}\left[S_{m}\right]$. Then by the claim $n_{i, q}>\min \left\{n_{i}, q\right\}=n_{i}$ for all $i \in$ $\{1, \ldots, m\}$. Hence $M_{q}=M$ which completes the proof of the Theorem.

The result in this Theorem is also the best one can obtain by only looking at the number of different composition factors in a composition series of a module of finite length as the following example shows.

Let $A=Z$ be the ring of integers and let $a=p_{1}^{n_{1}} \ldots p_{m}^{n_{m}}$, where $p_{1}, \ldots, p_{m}$ are different primes. Consider $A /(a)$. Then $[A /(a)]=n_{1}\left[Z / p_{1} Z\right]+\cdots+n_{m}\left[Z / p_{m} Z\right]$ and $\operatorname{LL}(\operatorname{End}(A /(a)))=\max \left\{n_{i} \mid i=1, \ldots, m\right\}$.

\section{REFERENCES}

1. F. W. Anderson and K. R. Fuller, Rings and categories of modules, Graduate Texts in Math., vol. 13, Springer-Verlag, New York, 1974.

Matematisk Institutt, Universitetet I Trondhem NLHT, 7055 Dragvoll, Norway

Current address: Forschungsinstitut für Mathematik, ETH-Zentrum, CH-8092 Zürich 